\title{
Liver transplantation in children
}

\section{Deirdre Kelly*}

Pediatric liver transplantation (LT) is now routine treatment for children dying of end-stage liver failure in both developed and developing countries. ${ }^{1}$ The indications for transplantation include acute and chronic liver failure, inherited metabolic liver disease and unresectable hepatic tumors. The major indication worldwide is in children with biliary atresia who have had an unsuccessful Kasai portoenterostomy, accounting for more than $50 \%$ children transplanted under the age of 2 years in Europe and the USA. ${ }^{2}$

Fulminant hepatic failure secondary to viral hepatitis or poisoning is the main indication for acute liver failure. ${ }^{2}$

The significant increase in the survival rate throughout the 1990's in most centers has been sustained with most reporting 1-year actuarial survival rates > 90\% in elective patients and $>70 \%$ in acute liver failure. Long-term survival figures for $10-15$ years are $>$ $80 \% .^{3}$ Many aspects have contributed to improved survival in children post LT. These include improved preoperative management such as the treatment of hepatic complications and the importance of intensive nutritional support. ${ }^{4}$ The development of better preservation solutions and surgical techniques, such as reduction hepatectomy, split LT and the introduction of living related $\mathrm{LT}$, has extended $\mathrm{LT}$ to infants under the age of 1 year and weighing less than $10 \mathrm{~kg}$, which has effectively reduced the waiting list mortality from 25 to $5 \% .{ }^{5}$ Surgical complications have reduced, but sepsis and rejection remain significant issues.

The development of effective methods of diagnosis, prophylaxis and treatment of cytomegalovirus (CMV) with ganciclovir or valganciclovir means that these diseases are no longer a significant cause of mortality but morbidity remains high. ${ }^{6}$ In contrast, the absence of specific therapy for Epstein-Barr virus (EBV) means that infection rates are high in those children who are EBV negative pre transplant, and who receive an adult EBV positive liver. The development of molecular genetic diagnosis for EBV (polymerase chain reaction) means that progressive disease, or post-transplant lymphoproliferative disease (PTLD) may be prevented by preemptive reduction of immunosuppression in response to rising EBV titers. Treatment of established disease is now possible with rituximab or adoptive T-cell therapy. ${ }^{7}$

Furthermore, the incidence of acute and chronic rejection has fallen following the development of newer immunosuppressive drugs, which are more easily absorbed such as cyclosporin microemulsion (Neoral) or more potent such as tacrolimus. ${ }^{8}$ The use of induction immunosuppression with monoclonal antibodies (IL-2 inhibitors) such as daclizumab, a humanized antibody and basiliximab, a chimeric antibody with transfer to maintenance immunosuppression with renal sparing drugs such as mycophenolate mofetil or sirolimus (rapamycin) may reduce long-term renal dysfunction. ${ }^{9}$

A more recent approach is the development of steroid-free immunosuppressive regimes, which reduces hypertension, stunting and the cosmetic side effects of steroid therapy. It is effective in the short term but may be associated with an increase in the development of de novo autoimmune hepatitis in 2-3\% of children. ${ }^{10}$ This form of graft dysfunction is associated with an increasing incidence of non-specific autoantibodies (ANA, SMA and rarely LKM), graft hepatitis and elevated immunoglobulins and may be related to the progressive development of graft hepatitis with fibrosis. ${ }^{11}$

As long-term survival increases, attention has now focused on the quality of life achieved by children undergoing transplantation. It is clear that survivors have nutritional and endocrine rehabilitation, undergo puberty normally and are fertile, with several reports of successful pregnancy. Although

* Professor of Pediatric Hepatology, Birmingham Children's Hospital, Birmingham, England. University of Birmingham, Edgbaston, Birmingham, England.

Professor Kelly declares that she has received educational grants for clinical trial support from Novartis, Roche and Astellas.

Suggested citation: Kelly D. Liver transplantation in children. J Pediatr (Rio J). 2008;84(5):381-382.

doi:10.2223/JPED.1833 
there is clear maintenance of psychosocial development both physically and intellectually, recent studies suggest there may be deficits in cognitive functioning which may be related to long-term immunosuppression. ${ }^{12}$

The success of pediatric LT in the USA and Europe has increased the awareness and highlighted the need for such procedures in other parts of the world. The article by Mesquita et al. ${ }^{13}$ is an important example of the success of LT in children in Brazil in a new program. The study includes 84 children under the age of 18 years who were assessed and/or listed for LT over a 9-year period and reports excellent results in line with survival rates from other international centers. Most children were either transplanted for biliary atresia or fulminant hepatitis as reported from other centers.

There are some important differences however. Children waited for a long time on the waiting list (between 2 to 6 years in three cases) before dying of their disease. In Europe, children would rarely be placed on the list so early (or be expected to survive so long), so this reflects the difficulty in obtaining suitable donors for these children and the concern of their physicians that they have the opportunity for transplantation, by listing them early.

It is also surprising that despite the high mortality, long waiting list time and the scarcity of donors that the unit rarely used reduction hepatectomy (10/40), live-related or split LT $(1 / 40)$ to address these issues, but this may reflect the early experience of the unit and explains the relatively high median age of transplantation (6.6 years) as most children received whole grafts.

Nevertheless, there were similar rates of technical complications compared to other series but a low rate of retransplantation (2/40), which was also related to the lack of donors.

Survival figures for chronic elective transplantation were very satisfactory, but the survival following transplantation for fulminant liver failure was less than is expected elsewhere and demonstrates how challenging this particular condition is to manage medically and surgically.

These are encouraging data and the unit is right to be proud of their results. They need now to build on this excellent start, to follow up their survivors and document their long-term outcome with regard to quality of life, renal and graft function, and to consider innovative methods of circumventing the donor scarcity including consideration of live-related LT.

\section{References}

1. Kelly DA. Current results and evolving indications for liver transplantation in children. J Pediatr Gastroenterol Nutr. 1998; 27:214-21.

2. Busuttil RW, Farmer DG, Yersiz H, Hiatt JR, McDiarmid SV, Goldstein LI, et al. Analysis of long-term outcomes of 3200 liver transplantations over two decades: a single-center experience. Ann Surg. 2005;241:905-16.

3. Soltys KA, Mazariegos GV, Squires RH, Sindhi RK, Anand R, SPLIT Research Group. Late graft loss or death in pediatric liver transplantation: An analysis of the SPLIT database. Am J Transplant. 2007; 7:2165-71.

4. Kelly DA. Nutritional factors affecting growth before and after liver transplantation. Pediatr Transplant. 1997;1:80-4.

5. de Ville de Goyet J, Hausleithner V, Reding R, Lerut J, Janseen M, Otte JB. Impact of innovative techniques on the waiting list and results in pediatric liver transplantation. Transplantation. 1993; $56: 1130-6$

6. Posfay-Barbe KM. Optimal approach to prevent CMV disease. Pediatr Transplant. 2008;12:385-7.

7. Haque T, Wilkie GM, Taylor C, Amlot PL, Murad P, Iley A, et al. Treatment of Epstein-Barr-virus-positive post-transplantation lymphoproliferative disease with partly HLA-matched allogeneic cytotoxic T cells. Lancet. 2002;360:436-42.

8. Kelly D, Jara P, Rodek B, Lykavieris P, Burdelski M, Becker M, et al. Tacrolimus and steroids versus ciclosporin microemulsion, steroids, and azathioprine in children undergoing liver transplantation: randomised European multicentre trial. Lancet. 2004;364:1054-61.

9. Kelly DA. Current issues in pediatric transplantation. Pediatr Transplant. 2006;10:712-20.

10. Venick RS, McDiarmid SV, Farmer DG, Gornbein J, Martin MG, Vargas $\mathrm{JH}$, et al. Rejection and steroid dependence: unique risk factors in the development of pediatric post transplant de novo autoimmune hepatitis. Am J Transplant. 2007;7:955-63.

11. Evans HM, McKiernan PJ, Beath SV, de ville de Goyet J, Kelly DA. Histology of liver allografts following paediatric liver transplantation. J Pediatr Gastroenterol Nutr. 2001;23:383.

12. Bucuvalas JC, Alonso E. Long-term outcomes after liver transplantation in children. Curr Opin Organ Transplant. 2008; 13:247-51.

13. Mesquita MC, Ferreira AR, Veloso LF, Roquete ML, de Lima AS, Pimenta JR, et al. Pediatric liver transplantation: tem years of experience at a single center in Brazil. J Pediatr (Rio J). 2008; 84: 395-402

Correspondence:

Deirdre A. Kelly

Birmingham Children's Hospital

Birmingham B4 6NH

University of Birmingham

Edgbaston, Birmingham - England

Tel.: +44 (12) 1333.8253

E-mail: deirdre.kelly@bch.nhs.uk 